\title{
Cognitive Behavioral Treatment for Posttraumatic Stress Disorder in Children and Adolescents
}

\author{
Shannon Dorsey, \\ University of Washington School of Medicine \\ Ernestine C. Briggs, and \\ Duke University School of Medicine \\ Briana A. Woods \\ University of North Carolina at Chapel Hill, Gillings School of Global Public Health
}

\begin{abstract}
A number of Cognitive Behavioral Therapy (CBT) approaches are available for treating child and adolescent posttraumatic stress disorder (PTSD). Similar to other CBT treatments, particularly those for anxiety disorders, these treatments all include common elements (e.g., psychoeducation, relaxation and affective modulation skills, exposure). The goals of this review are to: 1) delineate common elements in CBT approaches for treating child and adolescent PTSD; 2) provide a detailed review of two CBT approaches with substantial evidence of effectiveness; and 3) describe "Promising Practices," in the area of CBT approaches to treating child and adolescent PTSD. The two treatments reviewed in detail are Trauma-focused Cognitive Behavioral Therapy (TF-CBT) and Cognitive Behavioral Intervention for Trauma in Schools (CBITS). For these treatments, we describe the research evidence to date, specific elements of the treatment model, and discuss implementation and cultural considerations. In the "Promising Practices" section, other CBT approaches are reviewed that include many of the common elements; however, these approaches have accumulated less evidence of effectiveness to date. Research on CBT approaches to treating PTSD is ongoing, with a growing focus on explicit consideration of the multiple systems in which youth exposed to trauma are involved, and ways to better address co-occurring difficulties (e.g. serious behavior problems, substance use). Future directions for the field are discussed. These include further study of promising practices, cultural applicability of CBT approaches to treating PTSD, and strategies to enhance implementation and dissemination efforts to improve access to high-quality, evidence-based care for children and adolescents with PTSD.
\end{abstract}

\section{Keywords}

PTSD; children; adolescents; cognitive behavioral; treatment

Rates of exposure to violence and traumatic events for children and adolescents are exceedingly high. In a nationally representative sample of children and adolescents in the

(c) 2011 Elsevier Inc. All rights reserved.

Corresponding Author: Shannon Dorsey, PhD; dorsey2@uw.edu; Division of Public Behavioral Health and Justice Policy; University of Washington, School of Medicine; 2815 Eastlake Avenue East, Suite 200; Seattle, WA 98102; ph.: 206.685.6398; fax: 206.685.3430.

Publisher's Disclaimer: This is a PDF file of an unedited manuscript that has been accepted for publication. As a service to our customers we are providing this early version of the manuscript. The manuscript will undergo copyediting, typesetting, and review of the resulting proof before it is published in its final citable form. Please note that during the production process errors may be discovered which could affect the content, and all legal disclaimers that apply to the journal pertain. 
United States, $60.4 \%$ reported exposure in the past year, with lifetime rates nearly a half to one-third higher, depending on exposure type (Finkelhor, Turner, Omrod, \& Hamby, 2009). Many children and adolescents experience repeated exposure or multiple types of events over their lifetime (Copeland, Keeler, Angold, \& Costello, 2007; Finkelhor et al., 2009). Rates of trauma exposure for youth in war-involved or high-conflict countries are even higher (e.g., Derluyn, Broekaert, Schuyten, \& De Temmerman, 2004). The range of potentially traumatic events includes exposure to domestic violence, child abuse and neglect, community violence, and experiencing the violent death of a loved one, among others.

A significant number of children and adolescents exposed to traumatic events develop Posttraumatic Stress (PTS) symptoms, Posttraumatic Stress Disorder (PTSD), and other common trauma-related sequelae including depressive disorders, anxiety disorders, and externalizing behavioral disorders. Rates of PTSD among children and adolescents vary, depending on the study population of focus (i.e., traumatized sample vs. community sample), and particular type of trauma examined (i.e., sexual abuse and extreme interpersonal trauma are associated with higher rates of PTSD). According to recent studies; however, even subclinical symptoms of PTSD place children at risk for other psychiatric disorders (Copeland et al., 2007). Therefore, it is critical that children and adolescents receive effective treatment for PTS, PTSD, and co-occurring conditions (American Academy of Child and Adolescent Psychiatry, 2010).

Treatments with evidence of effectiveness for child and adolescent PTSD are available, the majority of which are Cognitive Behavioral Therapies (CBT; Chaffin \& Friedrich, 2004; Wetherington et al., 2008). In Wetherington and colleagues' (2008) meta-analytic review examining an array of treatment approaches for treating child and adolescent PTSD, the following approaches were reviewed: CBT, play therapy, art therapy, psychodynamic therapy, and pharmacologic therapy. The results were robust for CBT, whereas insufficient evidence was found for the other approaches. Silverman et al. (2008) provide further evidence for CBT approaches. In their review of psychosocial treatments for trauma exposure that have evidence for improving child and adolescent outcomes (i.e., posttraumatic stress, depressive symptoms, anxiety symptoms, and externalizing behavior problems), the only two that met the well-established and probably efficacious criteria (Chambless et al., 1996; Chambless \& Hollon, 1998) were both CBT approaches (i.e., Trauma-focused Cognitive Behavioral Therapy [TF-CBT] and Cognitive Behavioral Intervention for Trauma in Schools [CBITS]).

The available CBT approaches for PTSD have a number of common elements, many of which are also prevalent in most CBT treatments for other internalizing disorders (e.g., other anxiety, depression; Chorpita, 2006; Chorpita, Bernstein, \& Daleiden, 2008). These elements include: 1) Psychoeducation about PTSD, anxiety, and the prevalence and impact of trauma; 2) Relaxation and Affective Modulation Skills for managing physiological and emotional stress; 3) Exposure or Gradual Desensitization to memories of the traumatic event and to innocuous reminders of the traumatic event, and 4) Cognitive Restructuring of inaccurate or maladaptive/unhelpful cognitions. In Chorpita and colleagues' work identifying core components in the treatment of anxiety disorders, exposure appears to be the only "universal' component (Chorpita, Dalieden, \& Weisz, 2005). Exposure is explicitly included in the two trauma-specific CBT approaches with the most evidence (i.e., TF-CBT, CBITS), but is not always an explicit component of some of the promising practices included in this review.

In addition to these common clinical elements, CBT treatment approaches to PTSD also include common structural or delivery components, including agenda setting, modeling and coached practice of new skills in session, and assignment of weekly practice of skills in real- 
world settings (e.g., home, school), to occur in between sessions. Additional aspects of trauma-specific CBT include use of assessment measures to guide treatment, ongoing use of feedback, and progressively building on mastered skills. As in all CBT approaches, the therapist takes an active and directive role in session. In trauma-specific CBT, this role is particularly important, given that avoidance is one of the primary symptom areas of PTSD.

In this review, we provide a detailed overview of two CBT approaches with the strongest evidence of effectiveness: TF-CBT (Cohen, Mannarino, \& Deblinger, 2006) and CBITS (Stein et al., 2003). In addition to reviewing these two approaches, we include a "Promising Practices" section, in which we review a number of promising CBT approaches that contain many of the common elements listed above. The majority of these approaches are currently under investigation and merit attention, but have comparatively less evidence of effectiveness to date.

A number of investigators have reviewed psychosocial treatments for PTSD and trauma exposure (e.g., Chaffin \& Freidrich, 2004; Feeny, Foa, Treadwell, \& March, 2004; Silverman et al., 2008; Taylor \& Chemtob, 2004; Wetherington et al., 2008). This article provides an update to these reviews, a focus specifically on CBT approaches, and highlights selected promising practices. Moreover, evidence from the included promising practices both bolsters the evidence for CBT approaches to treating PTSD in children and adolescents in general and demonstrates the versatility and potential of CBT in varied settings and with diverse youth and families. In addition, many of the promising practices demonstrate that trauma-specific CBT approaches can be combined with other CBT interventions for treating PTSD as well as co-occurring disorders.

\section{Trauma-focused Cognitive Behavioral Therapy (TF-CBT)}

\section{Evidence}

Among the CBT approaches for trauma exposure, PTSD and co-occurring sequelae, TFCBT has the most evidence of effectiveness (Cohen,et al., 2006; Deblinger \& Heflin, 1996; www.musc.edu/tfcbt). To date, TF-CBT has six published randomized controlled trials (RCTs) supporting effectiveness in reducing PTS symptoms and PTSD, depressive symptoms, shame, and trauma-related and general behavior problems, in comparison to nonCBT interventions (e.g., supportive or client-centered therapies, waitlist control, usual care; Cohen \& Mannarino, 1996; Cohen \& Mannarino, 1998; Cohen, Deblinger, Mannarino, \& Steer, 2004; Deblinger, Lippmann, \& Steer, 1996; Deblinger, Stauffer, \& Steer, 2001; King et al., 2000). All RCTs except one (i.e., Deblinger et al., 2001) involved individual TF-CBT delivery. An additional small Randomized Controlled Trial (RCT) comparing TF-CBT alone to TF-CBT plus sertraline (Cohen, Mannarino, Perel, \& Staron, 2007) demonstrated little perceived benefit of added pharmacological intervention. Results from two additional RCTs are forthcoming, one of which focuses specifically on youth exposed to domestic violence (Cohen, Mannarino, \& Iyengar, in press) and one that examines variation in the number of sessions and aspects of gradual exposure (Deblinger, Mannarino, Cohen, Runyon \& Steer, in press). Follow-up studies provide evidence of sustained benefit at 6-months, one-year, and two-years posttreatment (Cohen \& Mannarino, 1997; Cohen, Mannarino \& Knudsen, 2005; Deblinger, Mannarino, Cohen, \& Steer, 2006; Deblinger, Steer, \& Lippmann, 1999). Trials have focused predominantly on school-age and preschool age youth who have been sexually abused or multiply traumatized.

In the most recently published multisite RCT involving 229 children ages $8-14$ years, all youth were sexually abused, with $90 \%$ of these youth experiencing a mean of 3.7 different types of traumatic events, including sexual abuse (Cohen et al., 2004). Children who received TF-CBT were half as likely as those in the client-centered comparison condition to 
meet full DSM-IV PTSD criteria at the end of treatment. Children in the TF-CBT condition also had significantly lower levels of depression and behavior problems as well as higher levels of interpersonal trust, perceived credibility and lower levels of shame. Parents of children who received TF-CBT also experienced improvement in depressive symptoms, parenting ability, and in their own abuse-related distress.

In addition to these RCTs, the evidence for TF-CBT is supplemented by a number of quasiexperimental (Hoagwood \& CATS Consortium, in press; Jaycox et al., 2010; Weiner, Schneider, \& Lyons, 2009) and open trials (e.g., Cohen, Mannarino, \& Knudsen, 2004; Cohen, Mannarino, \& Staron, 2006; Deblinger, McLeer, \& Henry, 1990; Stauffer \& Deblinger, 1996). Two open trials have focused specifically on childhood traumatic grief (Cohen et al., 2004; Cohen et al., 2006). One open trial provides additional evidence for group delivery of TF-CBT with sexually abused youth (Stauffer \& Deblinger, 1996).

\section{Model description}

The TF-CBT model includes nine components, described using the acronym, PRACTICE. The PRACTICE components include Psychoeducation, Parenting skills, Relaxation skills, Affective Modulation skills, Cognitive Coping skills, Trauma Narrative and Processing, In Vivo Exposure, Conjoint Child-Parent Sessions, and Enhancing Safety. TF-CBT is typically delivered in 12-20 sessions and is appropriate for children and adolescents ages 3 to 18 years (Cohen et al., 2006). In TF-CBT, the clinician works with both the child and the child's nonoffending caregiver, usually a biological parent. In the beginning of treatment, the sessions typically involve meeting individually with the child and the parent. The PRACTICE skills are taught to both, with the exception of Parenting, which is only taught to the parent. The goal of each component is to help the child and the parent achieve mastery over avoidance of trauma-related thoughts, feelings, reminders, and memories. The components are ordered in such a way that each component builds on the previous component, and therefore, the components are typically provided in the PRACTICE order, with early PRAC skillsbuilding components being delivered first.

A crucial part of providing TF-CBT involves the inclusion of exposure, or gradual exposure (GE) to feared stimuli. As previously mentioned, exposure has been identified as one of the common elements in CBT approaches for treating PTSD and other anxiety disorders. In the area of trauma, feared stimuli may include both memories and physical reminders of the trauma (e.g., sights, sounds, people, smells, other cues that serve as trauma reminders). Gradual exposure is integrated into all of the PRACTICE components, as it is a critical part of achieving mastery over avoidance. In each PRACTICE component, GE involves incrementally increasing the duration with which the child and the parent face feelings, thoughts, reminders and memories of the child's traumatic experience(s). The child can then habituate to the physical and psychological arousal that accompanies reminders of traumatic events such that avoidance and other symptoms are decreased. In addition to being included in all PRACTICE components, the Trauma Narrative portion of TF-CBT involves helping the child to gradually develop a narrative of the child's traumatic experiences that can be reviewed during subsequent sessions. During the Trauma Narrative component, which occurs over a number of sessions (e.g., 3-4), the child describes details of what happened before, during and after the traumatic event(s) as well as thoughts, feelings, and physiological reactions.

When the traumatic event involves death and loss, grief-specific components are available (CTGweb: http://ctg.musc.edu). These components include Grief-specific Psychoeducation, Grieving the Loss, Resolving Ambivalent Feelings, Redefining the Relationship (from interaction to memory), and Committing to Present Relationships. Each of these components 
builds systematically on the PRACTICE skills and can be tailored to meet the unique circumstances of children and adolescents.

\section{Implementation considerations}

A number of recent efforts demonstrate success in delivering TF-CBT with special populations of youth, including those in foster care, residential settings, and in international settings. Evidence of effectiveness with youth in foster care is accumulating, from the Weiner and colleagues study (2009) in Illinois and an ongoing National Institute for Mental Health (NIMH)-funded, randomized, effectiveness trial of TF-CBT in Washington State, focused on foster parent involvement and engagement (MH079910; Dorsey, PI).

Providing evidence for effectiveness in community-based settings, clinicians in many of the quasi-experimental and open trials of TF-CBT were masters-level clinicians employed in community mental health settings, and included youth who presented at mental health centers for treatment (i.e., Hoagwood \& CATS Consortium, in press; Jaycox et al., 2010; Weiner et al., 2009). Research on TF-CBT in a range of settings with varying implementation conditions is ongoing (for a review, see Dorsey \& Cohen, in press). Among these, a number of statewide implementation projects include relatively rigorous evaluation plans (e.g., Project BEST in South Carolina, www.musc.edu/projectbest; North Carolina Child Treatment Program in North Carolina, http://www.cfar.unc.edu/). Two NIMH-funded open trials of TF-CBT are currently underway in low-resource countries, one focused on HIV-infected children and adolescents who were sexually abused (i.e., Zambia) and one focused on orphaned children and adolescents, many of whom were orphaned as a result of the AIDS epidemic, who have traumatic grief symptoms (i.e., Tanzania).

\section{Cultural considerations}

In the United States, applications of TF-CBT have been developed for Latino (de Arellano et al., 2005) and Native American families (Bigfoot \& Braden, 2007). These applications maintain all of the TF-CBT components, but include culturally-specific aspects of each (e.g., Cuentos therapy - story-telling for Latino families) to better engage families and to ensure that the treatment is as culturally relevant as possible. In addition to these specific applications, all TF-CBT trainings, resources, and materials (e.g., Cohen et al., 2006, TFCBTWeb) specify the need for providing the model components with fidelity, but in a manner that is flexible and engaging with regard to family and child background, ethnicity, and culture. Internationally, TF-CBT is currently being implemented in a range of settings in Zambia, Tanzania, China, Japan, Norway, Cambodia, Indonesia, Germany, the Netherlands and other countries.

\section{Cognitive Behavioral Intervention for Trauma in Schools (CBITS)}

\section{Evidence}

To date, evidence for CBITS consists of one RCT (Stein et al., 2003), one quasiexperimental trial (Kataoka et al., 2003), and one field trial (Jaycox et al., 2010). In the RCT with sixth and seventh grade students $(N=126)$, Stein and colleagues (2003) evaluated CBITS compared to a waitlist control group. The intervention included 10 weekly, 45-60 minute group sessions, one to three individual sessions focused on imaginal exposure to the traumatic event(s), two to four optional sessions with parents, and one teacher education session. Following the 10-week CBITS intervention, the intervention group reported significantly lower PTSD symptoms compared to the waitlist control group, with $86 \%$ of students in the CBITS condition reporting lower PTSD symptom scores than would have been expected without treatment. In addition, the CBITS group reported lower depression scores, with $67 \%$ of students reporting lower depression scores than would have been 
expected without treatment. For both PTSD and depression scores, differences between the two groups disappeared after the waitlist delayed intervention group received CBITS. Further, 78\% of parents whose children received CBITS reported reduced psychosocial problems post-treatment; however, teachers did not report a significant reduction in classroom behavioral problems. The improvements in PTSD and depression symptoms, and parent-reported behavioral problems, were sustained at 6-month follow-up.

In their quasi-experimental study, Kataoka and colleagues (2003) evaluated CBITS, with Spanish-speaking, recent immigrant students $(N=113)$, also utilizing a waitlist comparison condition. Students recently immigrated (i.e., within the last three years) from Mexico (57\%), El Salvador (18\%), Guatemala (11\%) and other Latin American countries (13\%; Kataoka et al., 2003). Bilingual clinicians conducted eight weekly, 45-60 minute group sessions, one to three individual sessions with students, two to four optional sessions with parents, and one teacher education session. Specific emphasis was placed on culturally competent implementation in this study of CBITS. For example, support was provided for immigration-related loss and separation during parent sessions. Students in the CBITS condition with baseline PTSD symptoms in the clinical range showed a significant reduction in both PTSD and depression symptoms, compared to those in the waitlist condition.

In the field trial for Project Fleur-de-Lis (Jaycox et al., 2010), children who screened positive for PTSD symptoms 15-months post Hurricane Katrina were randomized to either a school-based group intervention (CBITS) or an individual intervention (TF-CBT) delivered at a mental health clinic. Overall, children in both intervention groups showed reductions in PTSD symptoms, although a number of children continued to have elevated symptoms posttreatment. CBITS appeared to be more accessible, however, with considerably more children beginning (98\%) and completing (91\%) treatment than in the TF-CBT condition, in which treatment was provided in a mental health clinic (23\% and $15 \%$, respectively).

\section{Model description}

CBITS was originally designed for trauma-impacted, recently immigrated students from Latino, Korean, Armenian, and Russian backgrounds, to be delivered in inner-city school mental health clinics (Stein et al., 2003). The developers utilized a participatory research framework that involved providers and family members in model design. CBITS targets youth between the ages of 10 and 15 and originally focused on exposure to community violence, but also has been found effective for youth with a range of trauma histories. Although developed for the school setting, CBITS has also been implemented in other settings (e.g., community mental health clinics).

CBITS, in a manner similar to TF-CBT, includes the common elements for trauma-specific CBT interventions for PTSD. CBITS incorporates psychoeducation, relaxation training, cognitive coping skills, gradual exposure to trauma memories through trauma narrative, in vivo exposure, affective modulation skills, cognitive restructuring, and social problem solving (Stein et al., 2003). CBITS is delivered in a group setting (6-8 children per group), and typically includes ten weekly sessions that are approximately one hour in length. In addition to the ten group sessions, there are one to three individual sessions focused on imaginal exposure to the traumatic event that occur before the gradual exposure exercises. CBITS also includes two parent education sessions where parents learn about the effects of trauma on youth and the skills the youth are learning in treatment. Lastly, CBITS includes one teacher education session in which teachers learn about the effects of trauma on youth and the ways trauma-related symptoms may present in the classroom (Stein et al., 2003).

An adaptation of CBITS, Support for Students Exposed to Trauma (SSET), was developed for delivery by school personnel (Jaycox, Langley, \& Dean, 2009). The adaption involved 
using a lesson plan format, eliminating individual break-out sessions and parent sessions, and using a more curricular format for imaginal exposure. A randomized trial of SSET $(N=$ 76), demonstrated reductions in PTSD and depressive symptoms, particularly for youth with high levels of symptoms pre-treatment (Jaycox et al., 2009). Compared to CBITS effects, smaller reductions in symptoms were seen with SSET.

\section{Implementation considerations}

CBITS aims to increase intervention "reach" (Zatzick, Koepsell; \& Rivara, 2009) by addressing common barriers to treatment such as stigma and access through providing treatment in the school setting. CBITS has been implemented in a variety of communities in the United States (e.g., immigrant, urban ethnic minority, low SES, middle class) and internationally (i.e., Australia, Japan; for more information, see, www.nctstnet.org; Treatments that Work). CBITS has been specifically adapted for the Latino immigrant faith community (Kataoka et al., 2006) and to be delivered by non-clinical school staff (i.e., SSET; Jaycox et al., 2009).

\section{Trauma and Grief Component Therapy (TGCT)}

\section{Evidence}

TGCT is a CBT-based treatment for youth (ages 12-20) who have been trauma-exposed or traumatically bereaved. TGCT has been primarily provided in schools, although it can be delivered in community mental health or other service settings. Evidence for TGCT comes from one RCT (Layne et al., 2008), two quasi-experimental studies (Goenijian et al., 1997; Hoagwood \& CATS Consortium, in press) and two open trials (Layne et al., 2001; Saltzman, Pynoos, Layne, Steinberg, \& Aisenberg, 2001). Three of these studies were conducted in a low-resource, international setting (i.e., Bosnia).

In the RCT (Layne et al. 2008), 127 war-exposed Bosnian youth (ages 13-19) in 10 secondary schools were randomized to receive only a classroom-based psychoeducation and skills intervention (modules one and four of TGCT) or the classroom-based intervention and the 17-session, group TGCT intervention. Both groups had significant reductions in PTSD symptoms at end of treatment and a four-month follow-up. For youth who experienced at least one bereavement, a subgroup of those enrolled in both conditions, only those in the TGCT group had reductions in grief symptoms (at end of treatment, grief symptoms were not assessed at the four-month follow-up). A significant decrease in depressive symptoms was demonstrated for both groups at the four-month follow-up, but only for the TGCT group at the end of treatment. Improvements for PTSD and depression were greater for those in the TGCT group than for those in the classroom-based intervention.

In the most recent quasi-experimental study, the Child and Adolescent Treatment Services (CATS; Hoagwood \& CATS Consortium, in press) project, which provided traumatreatment services to children and adolescents in New York City post 9-11, over half of the youth who received TGCT demonstrated reduced PTSD symptoms at end of treatment, with an even greater percentage demonstrating improvement at a 4-month follow up. In a quasiexperimental study of TGCT in Armenia ( $N=64$; Goenijian et al., 1997), receipt of TGCT was associated with reduced PTSD symptoms and stabilization of depressive symptoms, compared to youth who did not receive TGCT (and for whom depressive symptoms worsened). In the open trial conducted in the United States ( $N=26$; Saltzman et al., 2001), youth who received TGCT had decreased symptoms of PTSD and traumatic grief, if traumatically bereaved. In a open trial conducted in Bosnia ( $N=55$; Layne et al., 2001), similar outcomes were demonstrated for PTSD and traumatic grief, in addition to decreased depressive symptoms. Interestingly, however, nearly half of the 55 youth $(n=27)$ received 
only the first two of four modules (i.e., did not receive the third grief-specific module; see below), yet evidenced similar reductions in PTSD and traumatic grief.

\section{Model Description}

TGCT is typically group-based and includes approximately 10-24 sessions corresponding with four modules. The goal of the first module is to reduce acute distress and build group cohesion. Module one includes common CBT elements of psychoeducation, and relaxation, among other skills for dealing with distress. Module two involves gradual exposure and cognitive processing. Module three involves providing grief-specific components (e.g., psychoeducation about grief, adaptive remembering of the deceased). In the fourth module, the focus is on moving forward, and includes problem-solving current difficulties, additional restructuring of maladaptive cognitions (e.g., core beliefs), and taking steps towards restoring normal developmental progression.

\section{Implementation considerations}

TGCT has been delivered in both the United States with diverse populations and in a lowresource setting. Providers of TGCT have included "real-world" providers, both in the domestic and international studies, indicating feasibility in community-based settings with community providers. In addition, in two of the studies (Layne et al., 2001; Layne et al., 2008), groups demonstrated improvement in PTSD symptoms despite receiving only part of TGCT, suggesting further research attention to the potential benefit of more limited duration or limited complexity of interventions for traumatized youth.

\section{Promising Practices}

A number of CBT "promising practices" for treating PTSD are available and merit review. Many of these treatments are currently being evaluated, and as research continues to accumulate, these treatments may supplement our menu of options and understanding of how to effectively treat children and adolescents with PTSD. Many of these practices combine common elements of CBT approaches to treating trauma with other CBT interventions, or aspects of other interventions to explicitly address system-related issues or common comorbid conditions (e.g., substance use, emotion dysregulation, depression). The treatment developers of many of these practices have received additional resources and support as part of the congressionally-mandated National Child Traumatic Stress Network (NCTSN; www.nctsn.org) that is administered through the Substance Abuse Mental Health Services Administration (SAMHSA). The NCTSN is a science to practice collaborative of more than 50 currently-funded centers that combines resources from hospitals, universities, community-based organizations, schools, child welfare organizations, and other entities committed to increasing access to and raising the standard of care for children, adolescents, and their families affected by trauma. As such, the NCTSN has been a major catalyst for the development, implementation, and dissemination of a variety of evidence-based and promising practices. In the section below, we review a few of these promising practices. Notably, many specifically target youth with chronic trauma exposure and high levels of emotional dysregulation.

\section{Trauma Systems Therapy (TST)}

Inspired by Bronfenbrenner's social-ecological model (Bronfenbrenner, 1979), TST is a systems-oriented, CBT-informed treatment for trauma-exposed youth that focuses on both PTSD and other trauma-related symptoms and on explicitly attempting to remediate factors in the social environment that perpetuate symptoms (for more information, see Saxe, Ellis, \& Kaplow, 2007). Evidence to support the efficacy of TST comes from an open trial of 110 youth, ages 5-20 (Saxe, Ellis, Fogler, Hansen, \& Sorkin, 2005). Participants demonstrated 
significant improvements in PTSD symptoms and family and school-related problems over a three-month follow-up period.

TST utilizes a multi-disciplinary team to implement an array of interventions, within multiple systems, that target two key dimensions: (1) enhancing individuals' ability to regulate emotions and cope with considerable adversity; and (2) promoting change in the social environment (e.g., increasing safety in the home, obtaining adequate housing) to decrease ongoing stress and threats that the child experiences. TST includes five phases (i.e., surviving, stabilizing, enduring, understanding, and transcending) which include many of the common CBT elements (e.g., affective modulation, cognitive restructuring) as well as interventions in the broader systems designed to stabilize the child, family, and/or distressed and threatening social environments. For example, TST may include legal advocacy, case management, care coordination and psychopharmacological interventions. TST utilizes structured assessments to determine which phase of treatment is indicated. This phase-based treatment is particularly useful for families who encounter a range of barriers to treatment engagement, multiple traumas, and a host of social environment issues.

\section{Combined Parent Child Cognitive-Behavioral Approach for Children and Families At-Risk for Child Physical Abuse (CPC-CBT)}

CPC-CBT is a multi-family group intervention, designed for families at risk for, or who have committed, child physical abuse. In comparison to Kolko's Alternatives for Families model (AF-CBT; Kolko, 1996; Kolko \& Swenson, 2002), an existing Evidence-Based Practice (EBP) for physically abusive families, CPC-CBT specifically includes child PTSD as one of its primary treatment targets. Evidence for CPC-CBT comes from one RCT and one small open trial. Runyon, Deblinger, and Steer (2010) conducted a small RCT comparing CPC-CBT to parent-only CBT with 44 parents and their 60 children ( $7-13$ years old). Children and families who participated in the CPC-CBT demonstrated significant improvements in PTS symptoms and parents demonstrated greater improvements in positive parenting practices. The parent-only CBT treatment group; however, demonstrated greater reductions in the use of corporal punishment. In an open trial of CPC-CBT involving 12 families and their 21 children (ages 4-14) that preceded the RCT, child PTS symptoms improved and child and parent-reported physically abusive behavior decreased (Runyon, Deblinger \& Schroeder, 2009). Improvements were also seen in parenting consistency and parental anger towards the children.

CPC-CBT specifically targets PTSD, depression, abuse-related attributions, and externalizing behavioral problems in children and adolescents with a history of harsh physical discipline and/or coercive parenting strategies. CPC-CBT includes a treatment protocol for children and families at risk for child physical abuse that is built on TF-CBT, motivational interviewing, ancillary support services (e.g., babysitting, transportation), case management, and relationship enhancement skills that specifically target family violence (e.g., domestic violence). Parents and children attend 16 weeks of two-hour sessions. Parent and child interventions are conducted concurrently for the first 75 minutes of the session by two group therapists in each group. The second 45 minutes involves the integrated joint parent-child sessions.

\section{Structured Psychotherapy for Adolescents Responding to Chronic Stress (SPARCS)}

SPARCS is a 16-session group intervention designed specifically to address the needs of chronically traumatized adolescents ages $12-19$, living in or returning to chaotic environments and who may also be experiencing PTSD and problems in several domains of functioning (e.g., interpersonal problems, affect regulation and impulsivity, and selfperception; for more information, see DeRosa \& Pelcovitz, 2009). Evidence for SPARCS 
comes from a recent quasi-experimental trial implemented with 33 culturally diverse 13-21 year olds, who had experienced a moderate or severe, discrete traumatic experience (Weiner, Schneider, \& Lyons, 2009). The majority of participants were female (63.6\%) and ethnic minority (67\% African American; 12\% Latino; $21 \%$ White). In this study, SPARCS was found effective in improving traumatic stress symptoms, life domain functioning, and risk behaviors, but only for African American participants, perhaps due to insufficient sample size of the other two racial groups

Given that chronic trauma exposure disrupts the progression of many basic developmental tasks, the overarching goals of SPARCS include helping youth to deal with more complex forms of PTSD by: coping more effectively with trauma and related sequelae; enhancing self-efficacy; connecting with others and establishing supportive relationships; cultivating awareness; and creating meaning. The theoretical basis for SPARCS is predominantly CBT and includes many of the common elements noted above (e.g., psychoeducation, relaxation and affective modulation, cognitive processing). SPARCS also includes modules and interventions from Dialectical Behavior Therapy for Adolescents (e.g., mindfulness; Miller, Rathus, \& Linehan, 2007), Trauma Adaptive Recovery Group Education and Therapy for Adolescents and Pre-Adolescents (reviewed below), and TGCT. Notably, SPARCS does not include a formal exposure component; however, participants may choose to discuss and process traumatic experiences in the group with the guidance of the group facilitator.

\section{Trauma- Focused Coping (TFC; aka Multimodality Trauma Treatment, MMTT)}

Delivered in schools and considered a precursor to CBITS, TFC is a group-based CBT approach for children and adolescents exposed to single incident traumatic stressors (e.g., disaster, exposure to violence, murder, suicide, fire, accidents; for more information see Amaya-Jackson et al., 2003). Evidence to support the effectiveness of TFC comes from a single case, cross-setting design involving 17 participants (March, Amaya-Jackson, Murray $\&$ Schulte, 1998) and a small clinic-based open trial involving 7 youth (Amaya-Jackson et al., 2003). In the March and colleagues study (1998), students who received TFC reported reduced PTSD symptoms at the end of treatment and at follow-up as well as improved depression, anxiety, and anger. Youth with complex trauma presentations participated in the clinic-based open trial (Amaya-Jackson et al., 2003), which included both individual provision of TFC $(n=4)$ and group provision of TFC $(n=3)$. Youth had reduced PTSD symptoms post-treatment, but reductions were less robust than those found in the March et al. (1998) study.

TFC targets PTSD and collateral symptoms of depression, anxiety, anger, and external locus of control. It was designed as a peer-mediating and skill building group intervention for youth in late elementary school through middle school. TFC includes 14-18 group sessions delivered during one class period each week. An individual pullout session is done midprotocol to introduce narrative exposure in a controlled way.

\section{Risk Reduction through Family Therapy (RRFT)}

RRFT is a family-focused, integrated treatment that combines TF-CBT, Multisystemic Therapy (MST; Henggeler, Clingempeel, Brondino, \& Pickrel, 2002), and other approaches to reduce risky behavior (e.g., substance use, sexual risk behavior) and trauma-related symptoms among sexually-assaulted youth (Danielson et al., 2010). The seven primary targets of RRFT include: psychoeducation, coping, family communication, substance abuse, PTSD, healthy dating and sexual decision making, and revictimization risk reduction. In general, sessions are 60 to 90 minutes once a week with phone check-ins as needed and the number of sessions depend upon the youth's symptom level. A small open trial of RRFT ( $N$ $=10$ ) with adolescent females (ages 14-17) demonstrated reductions in PTSD and 
depression symptoms, reductions in substance use, slight improvements in family conflict and cohesion, and improvements in ecological functioning (e.g., spending time with prosocial peers, engaging in positive family activities, attending school/work). Treatment gains were maintained at 6-month follow-up.

\section{Trauma Adaptive Recovery Group Education and Therapy for Adolescents and Pre- Adolescents (TARGET-A)}

TARGET-A is an intervention for youth between the ages of 12-19 who have experienced interpersonal trauma (e.g., maltreatment) and an array of chronic trauma (e.g., domestic violence, community violence) and other stressors. TARGET-A utilizes some CBT skills (e.g., affective modulation skills, cognitive processing) in addition to other interventions. TARGET-A is usually delivered in 12 approximately 50-minute individual sessions. TARGET-A includes sequential skill-development modules designed to assist the adolescent in managing and preventing current PTSD symptoms (see Ford \& Russo, 2006 for more information). As in SPARCS, in TARGET-A, youth may choose to talk about past traumatic events (e.g., a part of gradual exposure, one of the common elements of trauma-specific CBT) but gradual exposure is not a core component of TARGET-A. The set of seven TARGET-A skills (i.e., FREEDOM skills) was specifically designed to address complex presentations of PTSD, and to focus on, and be delivered with delinquent and juvenile justice-involved youth. Findings from a recently completed RCT focused on girls (ages 13-17; N = 59) involved in delinquency are forthcoming (Ford, Steinberg, Hawke, Levine, $\&$ Zhang, 2010). Evidence for TARGET-A also comes from an RCT $(N=213)$ of TARGET with substance-using adults in an outpatient setting (Frisman, Ford, Lin, Mallon, \& Chang, 2008). TARGET was more effective in improving sobriety and PTSD related outcomes, but differences in effectiveness varied by ethnicity of the participants.

\section{Cultural Considerations}

In the area of child and adolescent PTSD treatment, as in other areas, the relevance of EBPs, many of which were CBT approaches, to culturally diverse populations has been the subject of much debate. This is due in large part to the disproportionate use of European American, middle-class families in early treatment studies. Moreover, many questioned validity of the use of EBPs given the absence of data supporting use with ethnically diverse populations. However, due to the relatively recent development of many of these treatments, studies of trauma-specific CBT have included diverse samples in the RCTs, quasi-experimental studies, and open trials (TF-CBT, CPC-CBT,TGCT); were developed specifically to target multicultural/multi-lingual populations (CBITS; TST, TARGET-A, SPARCS), and have cultural-specific applications (TF-CBT).

In addition, a growing number of studies focused on the use of EBPs with ethnic minority youth have demonstrated that EBPs for many disorders, including PTSD, may be more efficacious than usual care for ethnic minority youth (Huey \& Polo, 2008; Miranda et al., 2005) and that EBPs and cultural competence and sensitivity may be more complementary than disparate (Whaley \& Davis, 2007). Despite these advances, many questions remain regarding treatment effects and outcomes with ethnically diverse youth, both for PTSD and other disorders. Do culturally-specific applications, for example, enhance treatment outcomes for ethnically diverse youth? Have these treatments been tested with diverse populations within the community-based settings, in which they are typically provided? As a result of these and other questions, the field is beginning to increase its focus on the impact of cultural influences on client engagement, the therapeutic relationship, symptom expression, and improvement. In the area of trauma-specific CBT approaches, researchers and treatment developers are examining culturally-specific aspects of common elements such as cognitive processing, exposure, and/or highlighting ways in which culture maybe a 
source of mastery, strength, and resilience (deArellano, Ko, Danielson, \& Sprague, 2008). The field is advancing - and researchers in the area of PTSD treatment are at the forefront, given that the role of culture in the treatment of trauma and traumatic stress is crucial. Specifically, culture often plays a role in treatment-seeking behavior, preferences about treatment, engagement in treatment, and in beliefs about why traumatic experiences happened and what is necessary for recovery and improvement (Cohen, Deblinger, Mannarino, \& de Arellano, 2001).

\section{Summary and Future Directions}

Trauma-specific CBT interventions for treating child and adolescent PTSD are available, and research both on existing and relatively new CBT approaches to treating PTSD continues to accumulate. CBT treatments for PTSD include both individual and group approaches; interventions included in this review demonstrated improvements in PTSD symptoms with treatment, and many of these gains were sustained over time (i.e., TF-CBT, CBITS, TGCT). Notably, most studies of CBT approaches to treating trauma also demonstrated improvements in other commonly co-occurring difficulties (e.g., depression, anxiety, behavior problems). These improvements are likely due in part to the differential developmental manifestations of PTSD as well as the overlap in common elements across CBT treatments that also address these co-occurring difficulties. Future directions for the field include: continuing to conduct research on promising practices and their ability to remediate PTSD; examining issues related to cultural applicability and responsiveness; ensuring effective implementation and dissemination (e.g., treatment reach); and sustaining treatment gains over time. Additional next steps may also include the examination of the core elements of treatments and their relevance to workforce development as increasingly more clinicians begin to embrace evidence-based treatments.

\section{Acknowledgments}

This work was supported by a grant R34-MH079910 (SD) from the National Institutes of Health.

\section{References}

Amaya-Jackson L, Reynolds V, Murray M, McCarthy G, Nelson A, Cherney M, et al. Cognitive behavioral treatment for pediatric posttraumatic stress disorder: Protocol and application in school and community settings. Cognitive and Behavioral Practice. 2003; 10:204-213.

American Academy of Child and Adolescent Psychiatry. Practice parameter for the assessment and treatment of children and adolescent with posttraumatic stress disorder. Journal of American Academy of Child and Adolescent Psychiatry. 2010; 49:414-430.

Bronfenbrenner U. Contexts of child rearing: Problems and prospects. American Psychologist. 1979; 34:844-850.

Chaffin M, Friedrich B. Evidence-based treatments in child abuse and neglect. Children and Youth Services Review. 2004; 26(11):1097-1113.

Chambless, D.; Gillis, M. Advances in cognitive-behavioral therapy. Vol. 2. Thousand Oaks, CA US: Sage Publications, Inc.; 1996. Cognitive therapy of anxiety disorders; p. 116-144.

Chambless D, Hollon S. Defining empirically supported therapies. Journal of Consulting and Clinical Psychology. 1998; 66(1):7-18. [PubMed: 9489259]

Chorpita, BF. Modular cognitive behavioral therapy for childhood anxiety disorders. New York, NY: Guilford Press; 2006.

Chorpita BF, Bernstein A, Daleiden EL. Driving with roadmaps and dashboards: Using information resources to structure the decision models in service organizations. Administration and Policy in Mental Health and Mental Health Services Research. 2008; 35(1-2):114-123. [PubMed: 17987376] 
Chorpita BF, Daleiden EL, Weisz JR. Modularity in the design and application of therapeutic interventions. Applied \& Preventive Psychology. 2005; 11(3):141-156.

Cohen JA, Deblinger E, Mannarino A, de Arellano MA. The importance of culture in treating abused and neglected children: An empirical review. Child Maltreatment. 2001; 6:148-157. [PubMed: 16705790]

Cohen JA, Deblinger E, Mannarino AP, Steer RA. A multisite, randomized controlled trial for children with sexual abuse-related PTSD symptoms. Journal of the American Academy of Child and Adolescent Psychiatry. 2004; 43:393-403. [PubMed: 15187799]

Cohen JA, Mannarino AP. A treatment outcome study for sexually abused preschool children: Initial findings. Journal of the American Academy of Child and Adolescent Psychiatry. 1996; 35:42-50. [PubMed: 8567611]

Cohen JA, Mannarino AP. A treatment study for sexually abused preschool children: Outcome during a one-year follow-up. Journal of the American Academy of Child and Adolescent Psychiatry. 1997; 36:1228-1235. [PubMed: 9291724]

Cohen JA, Mannarino AP. Factors that mediate treatment outcome of sexually abused preschool children: Six- and 12-month follow-up. Journal of the American Academy of Child and Adolescent Psychiatry. 1998; 37:44-51. [PubMed: 9444899]

Cohen, JA.; Mannarino, AP.; Deblinger, E. Treating trauma and traumatic grief in children and adolescents. New York: Guilford Press; 2006.

Cohen JA, Mannarino AP, Iyengar S. Community treatment of PTSD in children exposed to intimate partner violence: A randomized controlled trial. Archives of Pediatrics and Adolescent Medicine. in press.

Cohen JA, Mannarino AP, Knudsen K. Treating childhood traumatic grief: A pilot study. Journal of the American Academy of Child and Adolescent Psychiatry. 2004; 43:1225-1233. [PubMed: 15381889]

Cohen JA, Mannarino AP, Knudsen K. Treating sexually abused children: 1 year follow-up of a randomized controlled trial. Journal of Child Abuse and Neglect. 2005; 29:135-145.

Cohen JA, Mannarino AP, Perel JM, Staron V. A pilot randomized controlled trial of combined Trauma-Focused CBT and sertraline for childhood PTSD symptoms. Journal of the American Academy of Child \& Adolescent Psychiatry. 2007; 46(7):811-819. [PubMed: 17581445]

Cohen JA, Mannarino AP, Staron VR. A pilot study of modified cognitive-behavioral therapy for childhood traumatic grief (CBT-CTG). Journal of the American Academy of Child and Adolescent Psychiatry. 2006; 45:1465-1473. [PubMed: 17135992]

Copeland WE, Keeler G, Angold A, Costello J. Traumatic events and posttraumatic stress in childhood. Archives of General Psychiatry. 2007; 64:577-584. [PubMed: 17485609]

Danielson CK, McCart MR, de Arellano MA, Macdonald A, Doherty LS, Resnick HS. Risk reduction for substance use and trauma-related psychopathology in adolescent sexual assault victims: Findings from an open trial. Child Maltreatment. 2010; 15:261-268. [PubMed: 20534594]

de Arellano, MA.; Ko, SJ.; Danielson, CK.; Sprague, CM. Trauma-informed interventions:Clinical and research evidence and culture-specific information project. Los Angeles, CA \& Durham, NC: National Center for Child Traumatic Stress; 2008.

Deblinger, E.; Heflin, AH. Treating sexually abused children and their nonoffending parents: A cognitive behavioral approach. Thousand Oaks, CA: Sage Publications; 1996.

Deblinger E, Lippmann J, Steer RA. Sexually abused children suffering posttraumatic stress symptoms: Initial treatment outcome findings. Child Maltreatment. 1996; 1:310-321.

Deblinger E, Mannarino AP, Cohen JA, Runyon M, Steer R. Trauma focused cognitive behavioral therapy for children: Impact of the trauma narrative and treatment length. Depression and Anxiety. in press.

Deblinger E, Mannarino AP, Cohen JA, Steer RA. A follow-up study of a multisite, randomized, controlled trial for children with sexual abuse-related PTSD symptoms. Journal of the American Academy of Child and Adolescent Psychiatry. 2006; 45:1474-1484. [PubMed: 17135993]

Deblinger E, McLeer S, Henry D. Cognitive behavioral treatment for sexually abused children suffering post-traumatic stress: Preliminary findings. Journal of the American Academy of Child \& Adolescent Psychiatry. 1990; 29:747-752. [PubMed: 2228928] 
Deblinger E, Stauffer LB, Steer RA. Comparative efficacies of supportive and cognitive behavioral group therapies for young children who have been sexually abused and their nonoffending mothers. Child Maltreatment. 2001; 6:332-343. [PubMed: 11675816]

Deblinger E, Steer RA, Lippman J. Two year follow-up study of cognitive behavioral therapy for sexually abused children suffering post-traumatic stress symptoms. Child Abuse and Neglect. 1999; 23:1371-1378. [PubMed: 10626618]

Derluyn I, Broekaert E, Schuyten G, De Temmerman E. Post-traumatic stress in former Ugandan child soldiers. Lancet. 2004; 363:861-863. [PubMed: 15031032]

DeRosa R, Habib M, Pelcovitz D, Rathus J, Sonnenklar J, Ford J, et al. Structured Psychotherapy for Adolescents Responding to Chronic Stress. 2006 Unpublished manual.

DeRosa, R.; Pelcovitz, D. Treating traumatized adolescent mothers: a structured approach. In: Webb, N., editor. Working with traumatized youth in child welfare. New York: Guilford Press; 2006. p. 219-245.

DeRosa, R.; Pelcovitz, D. Treating traumatized children: Risk, resilience and recovery. New York: Routledge/Taylor \& Francis Group; 2009. Group treatment for chronically traumatized adolescents: Igniting SPARCS of change; p. 225-239. Retrieved from PsycINFO database

Dorsey, S.; Cohen, J. Trauma-focused cognitive behavioral therapy. In: Clements, P.; Seedat, S., editors. Mental Health Issues of Child Maltreatment. Saint Louis, MO: GW Medical Publishing; in press

Feeny N, Foa E, Treadwell K, March J. Posttraumatic Stress Disorder in Youth: A Critical Review of the Cognitive and Behavioral Treatment Outcome Literature. Professional Psychology: Research and Practice. 2004; 35(5):466-476.

Finkelhor D, Turner H, Omrod R, Hamby SL. Violence, abuse, and crime exposure in a national sample of children and youth. Pediatrics. 2009; 124:1-13. [PubMed: 19564276]

Frisman L, Ford J, Lin H, Mallon S, Chang R. Outcomes of trauma treatment using the TARGET model. Journal of Groups in Addition and Recovery. 2008; 3(3-4):285-303.

Ford JD. Trauma, PTSD, and ethnoracial minorities: Toward diversity and cultural practices in principles and practices. Clinical Psychology: Science \& Practice. 2008; 15:62-67.

Ford JD, Russo E. Trauma-focused, present-centered, emotional self-regulation approach to integrated treatment for posttraumatic stress and addiction: Trauma Adaptive Recovery Group Education and Therapy (TARGET). American Journal of Psychotherapy. 2006; 60:335-355. [PubMed: 17340945]

Ford JD, Russo E, Mallon S. Integrating post-traumatic stress disorder (PTSD) and substance use disorder treatment. Journal of Counseling and Development. 2007; 85:475-489.

Ford JD, Steinberg KL, Hawke J, Levine J, Zhang W. Randomized Trial Comparison of Emotion Regulation and Relational Psychotherapies for PTSD with Girls Involved in Delinquency. 2010 Manuscript submitted for publication.

Henggeler S, Clingempeel W, Brondino M, Pickrel S. Four-year follow-up of multisystemic therapy with substance-abusing and substance-dependent juvenile offenders. Journal of the American Academy of Child \& Adolescent Psychiatry. 2002; 41(7):868-874. [PubMed: 12108813]

Hoagwood KE. CATS Consortium. Impact of CBT for traumatized children and adolescents affected by the World Trade Center disaster. Journal of Clinical Child and Adolescent Psychology. in press.

Huey SJ, Polo AJ. Evidence-based psychosocial treatments for ethnic minority youth. Journal of Clinical Child and Adolescent Psychology. 2008; 37:262-301. [PubMed: 18444061]

Jaycox, LH. Cognitive-behavioral intervention for trauma in schools. Longmont, CO: Sopris West Educational Services; 2003.

Jaycox LH, Cohen JA, Mannarino AP, Walker DW, Langley AK, Gegenheimer KL, et al. Children's mental health care following Hurricane Katrina: A field trial of trauma-focused psychotherapies. Journal of Traumatic Stress. 2010; 23:223-231. [PubMed: 20419730]

Jaycox, LH.; Langley, AK.; Dean, KL. Support for students exposed to trauma: The SSET program: group leader training manual, lesson plans, and lesson materials and worksheets. Santa Monica, CA: RAND Health; 2009. 
Jaycox LH, Langley AK, Stein BD, Wong M, Sharma P, Scott M, et al. Support for students exposed to trauma: A pilot study. School Mental Health. 2009; 1:49-60. [PubMed: 20811511]

Kataoka SH, Fuentes S, O'Donoghue VP, Castillo-Campos P, Bonilla A, Halsey K, et al. A community participatory research partnership: The development of a faith-based intervention for children exposed to violence. Ethnicity \& Disease. 2006; 16:89-97. [PubMed: 16599354]

Kataoka SH, Stein BD, Jaycox LH, Wong M, Escudero P, Tu W, et al. A school-based mental health program for traumatized Latino immigrant children. Journal of the American Academy of Child \& Adolescent Psychiatry. 2003; 42:311-318. [PubMed: 12595784]

King NJ, Tong BJ, Mullen P, Myerson N, Heyne D, Rollings S, et al. Treating sexually abused children with posttraumatic stress symptoms: A randomized clinical trial. Journal of the American Academy of Child and Adolescent Psychiatry. 2000; 39:1347-1355. [PubMed: 11068889]

Kolko D. Individual cognitive behavioral treatment and family therapy for physically abused children and their offending parents: A comparison of clinical outcomes. Child Maltreatment. 1996; 1(4): $322-342$.

Kolko, DJ.; Swenson, CC. Assessing and treating physically abused children and their families: A cognitive behavioral approach. Thousand Oaks, CA: Sage Publications; 2002.

Layne CM, Saltzman WR, Poppleton L, Burlingame GM, Pasalic A, Durakovic E, Music M, Campara N, Dapo N, Arslanagic B, Steinberg AM, Pynoos RS. Effectiveness of a school-based group psychotherapy program for war-exposed adolescents: A randomized controlled trial. Journal of the American Academy of Child and Adolescent Psychiatry. 2008; 47:1048-1062. [PubMed: 18664995]

Layne, CM.; Saltzman, WR.; Pynoos, RS.; Steinberg, AM. Trauma and Grief Component Therapy. New York: New York State Office of Mental Health; 2002.

Layne CM, Pynoos RS, Saltzman WR, Arslanagic B, Black M, Savjak N, et al. Trauma/grief-focused group psychotherapy: School-based postwar intervention with traumatized Bosnian adolescents. Group Dynamics: Theory, Research, and Practice. 2001; 5(4):277-290.

March J, Amaya-Jackson L, Murray M, Schulte A. Cognitive behavioral psychotherapy for children and adolescents with post-traumatic stress disorder following a single incident stressor. Journal of the American Academy of Child and Adolescent Psychiatry. 1998; 37(6):585-593. [PubMed: 9628078]

Miller, AL.; Rathus, JH.; Linehan, MM. Dialectical Behavior Therapy with suicidal adolescents. New York: Guilford Press; 2007.

Miranda J, Guillermo B, Lau A, Kohn L, Hwang WC, LaFramboise T. State of the science on psychosocial interventions for ethnic minorities. Annual Review of Clinical Psychology. 2005; $1: 113-142$.

Runyon MK, Deblinger D, Schroeder CM. Pilot evaluation of outcomes of combined parent-child cognitive-behavioral group therapy for families at-risk for child physical abuse. Cognitive and Behavioral Practice. 2009; 16:101-118.

Runyon MK, Deblinger D, Steer R. Comparison of combined parent-child and parent-only cognitivebehavioral treatments for offending parents and children in cases of child physical abuse. Child \& Family Behavior Therapy. 2010; 32:196-218.

Saltzman W, Pynoos R, Layne C, Steinberg A, Aisenberg E. Trauma- and grief-focused intervention for adolescents exposed to community violence: Results of a school-based screening and group treatment protocol. Group Dynamics: Theory, Research, and Practice. 2001; 5(4):291-303.

Saxe GN, Ellis BH, Fogler J. Comprehensive care for traumatized children: An open trial examines Trauma Systems Therapy. Psychiatric Annals. 2005; 35(5):443-448.

Saxe, GN.; Ellis, BH.; Kaplow, J. Collaborative care for traumatized children and teens: A Trauma Systems Therapy approach. Guilford Press; NY: 2006 October.

Silverman WK, Ortiz CD, Viswesvaran C, Burns BJ, Kolko DJ, Putnam FW, et al. Evidence-based psychosocial treatments for children and adolescents exposed to traumatic events. Journal of Clinical Child \& Adolescent Psychology. 2008; 37(1):156-183. [PubMed: 18444057]

Stauffer LB, Deblinger E. Cognitive behavioral Groups for nonoffending mothers and their young sexually abused children: A preliminary treatment outcome study. Child Maltreat. 1996; 1(1):6576. 
Stein BD, Jaycox LH, Kataoka SH, Wong M, Tu W, Elliott MN, Fink A. A mental health intervention for schoolchildren exposed to violence: A randomized controlled trial. Journal of the American Medical Association. 2003; 290:603-611. [PubMed: 12902363]

Taylor TL, Chemtob CM. Efficacy of treatment for child and adolescent traumatic stress. Archives of Pediatrics \& Adolescent Medicine. 2004; 158(8):786-791. [PubMed: 15289252]

Weiner DA, Schneider A, Lyons JS. Evidence-based treatments for trauma among culturally diverse foster care youth: Treatment retention and outcomes. Children and Youth Services Review. 2009; 31:1199-1205.

Wetherington HR, Hahn RA, Fuqua-Whitley DS, Sipe TA, Crosby AE, Johnson RL, et al. The effectiveness of interventions to reduce psychological harm from traumatic events among children and adolescents. American Journal of Preventive Medicine. 2008; 35(3):287-313. [PubMed: 18692745]

Whaley AL, Davis KE. Cultural competence and evidence-based practice in mental health services - A complementary perspective. American Psychologist. 2007; 62:563-574. [PubMed: 17874897]

Zatzick D, Koepsell T, Rivara F. Using target population specification, effect size, and reach to estimate and compare the population impact of two PTSD preventive interventions. Psychiatry: Interpersonal and Biological Processes. 2009; 72(4):346-359. 